Ferrata Storti Foundation

\title{
High-throughput elucidation of thrombus formation reveals sources of platelet function variability
}

\author{
Johanna P. van Geffen, ${ }^{1}$ Sanne L.N. Brouns, ${ }^{1}$ Joana Batista, ${ }^{2,3}$ Harriet \\ McKinney, ${ }^{2,3}$ Carly Kempster, ${ }^{2,3}$ Magdolna Nagy, ${ }^{1}$ Suthesh Sivapalaratnam, ${ }^{2,4}$ \\ Constance C.F.M.J. Baaten, ${ }^{1}$ Nikki Bourry, ${ }^{1}$ Mattia Frontini, ${ }^{2,3,5}$ Kerstin Jurk, ${ }^{6}$ \\ Manuela Krause, ${ }^{7}$ Daniele Pillitteri, ${ }^{7}$ Frauke Swieringa, ${ }^{1}$ Remco Verdoold, ${ }^{1}$ \\ Rachel Cavill, ${ }^{8}$ Marijke J. E. Kuijpers, ${ }^{1}$ Willem H. Ouwehand, ${ }^{2,3,5,9,10}$ Kate \\ Downes $^{2,3,9^{\star}}$ and Johan W.M. Heemskerk ${ }^{1^{*}}$
}

Volume 104(6):1256-1267

${ }^{1}$ Department of Biochemistry, Cardiovascular Research Institute Maastricht (CARIM), Maastricht University, the Netherlands; ${ }^{2}$ Department of Haematology, University of Cambridge, Cambridge Biomedical Campus, UK; ${ }^{3}$ National Health Service Blood and Transplant (NHSBT), Cambridge Biomedical Campus, UK; ${ }^{4}$ The Royal London Haemophilia Centre, London, UK; ${ }^{5} \mathrm{BHF}$ Centre of Excellence, Division of Cardiovascular Medicine, Cambridge University Hospitals, Cambridge Biomedical Campus, UK; ${ }^{6}$ Center for Thrombosis and Hemostasis (CTH), University Medical Center of the Johannes Gutenberg University Mainz, Germany; ${ }^{7}$ DKD Helios Klinik Wiesbaden, Germany; ${ }^{8}$ Department of Data Science \& Knowledge Engineering, Faculty of Humanities and Sciences, Maastricht University, the Netherlands; ${ }^{9}$ NIHR BioResource, University of Cambridge, Cambridge Biomedical Campus, UK and ${ }^{10}$ Department of Human Genetics, The Wellcome Sanger Institute, Hinxton, Cambridge, UK

*KD and JWMH contributed equally to this work.

\section{ABSTRACT}

\section{Correspondence:}

JOHAN W. M. HEEMSKERK

jwm.heemskerk@maastrichtuniversity.nl

KATE DOWNES

kd286@cam.ac.uk

Received: May 31, 2018.

Accepted: December 5, 2018.

Pre-published: December 13, 2018.

doi:10.3324/haematol.2018.198853

Check the online version for the most updated information on this article, online supplements, and information on authorship \& disclosures: www.haematologica.org/content/104/6/1256

\section{(C)2019 Ferrata Storti Foundation}

Material published in Haematologica is covered by copyright. All rights are reserved to the Ferrata Storti Foundation. Use of published material is allowed under the following terms and conditions:

https://creativecommons.org/licenses/by-nc/4.0/legalcode. Copies of published material are allowed for personal or internal use. Sharing published material for non-commercial purposes is subject to the following conditions:

https://creativecommons.org/licenses/by-nc/4.0/legalcode, sect. 3. Reproducing and sharing published material for commercial purposes is not allowed without permission in writing from the publisher.

\section{(c) (i) (s)}

I n combination with microspotting, whole-blood microfluidics can provide high-throughput information on multiple platelet functions in thrombus formation. Based on assessment of the inter- and intra-subject variability in parameters of microspot-based thrombus formation, we aimed to determine the platelet factors contributing to this variation. Blood samples from 94 genotyped healthy subjects were analyzed for conventional platelet phenotyping: i.e. hematologic parameters, platelet glycoprotein (GP) expression levels and activation markers (24 parameters). Furthermore, platelets were activated by ADP, CRP-XL or TRAP. Parallel samples were investigated for whole-blood thrombus formation ( 6 microspots, providing 48 parameters of adhesion, aggregation and activation). Microspots triggered platelet activation through GP Ib-V-IX, GPVI, CLEC-2 and integrins. For most thrombus parameters, inter-subject variation was 2-4 times higher than the intra-subject variation. Principal component analyses indicated coherence between the majority of parameters for the GPVI-dependent microspots, partly linked to hematologic parameters, and glycoprotein expression levels. Prediction models identified parameters per microspot that were linked to variation in agonist-induced $\alpha_{\text {II }} \beta_{3}$ activation and secretion. Common sequence variation of GP6 and FCER1G, associated with GPVI-induced $\alpha_{\text {II }} \beta_{3}$ activation and secretion, affected parameters of GPVIand CLEC-2-dependent thrombus formation. Subsequent analysis of blood samples from patients with Glanzmann thrombasthenia or storage pool disease revealed thrombus signatures of aggregation-dependent parameters that were subject-dependent, but not linked to GPVI activity. Taken together, this high-throughput elucidation of thrombus formation revealed patterns of inter-subject differences in platelet function, which were partly related to GPVI-induced activation and common genetic variance linked to GPVI, but also included a distinct platelet aggregation component. 


\section{Introduction}

Whole-blood-based microfluidics methods, measuring thrombus formation under flow, are increasingly used as proxy measurements for in vivo models of thrombosis. Such flow assays provide valuable mechanistic information on the consequences of loss-of-function or gain-offunction mutations of key platelet signaling proteins for arterial thrombosis and hemostasis. ${ }^{1.4}$ Until recently, collagen-coated surfaces were used for such measurements, so as to approximate common collagen-dependent models of arterial thrombosis in vivo., Recently, we showed that by using multiple, microspotted surfaces this method can be extended to multiparameter measurements of thrombus formation, ${ }^{7}$ thus elucidating platelet dysfunction in patients with a range of bleeding diatheses. ${ }^{7.9}$

Commonly, detection of an heritable or acquired platelet function impairment is made by conventional approaches, such as light transmission aggregometry, ${ }^{10,11}$ flow cytometry, ${ }^{12}$ and the PFA-100. ${ }^{13}$ However, such tests are limited by a low throughput and requirement of relatively large volumes of blood, if multiple agonists at various concentrations need to be tested. These limitations can be overcome by higher-throughput, well-plate-based tests of platelet aggregation or flow cytometric analysis. ${ }^{14,15}$ For the multiparameter measurement of thrombus formation, however, still little is known about the detection capability to identify (small differences in) platelet phenotypes in healthy subjects and groups of patients.

Two recent whole genome association studies have identified over 640 independent single nucleotide variants

Table 1. Overview of microspot surfaces $(M)$ and parameters $(P)$ in flow assays; as well as platelet activation $(A)$ markers in flow cytometry.

\begin{tabular}{llc} 
Microspot surface & Platelet receptors involved \\
$M 1$ & collagen type I (VWF)* & GPIb, GPVI, $\alpha_{2} \beta_{1}$ \\
$M 2$ & collagen type III (VWF)* & GPIb, GPVI, $\alpha_{2} \beta_{1}$ \\
\hline$M 3$ & VWF + laminin & GPIb $+\alpha_{6} \beta_{1}$ \\
$M 4$ & VWF-BP + GFOGER-(GPO) $)_{n}$ & $\mathrm{GPIb}+\mathrm{GPVI}, \alpha_{2} \beta_{1}$ \\
\hline$M 5$ & WWF-BP + rhodocytin & $\mathrm{GPIb}+\mathrm{CLEC}-2$ \\
$M 6$ & WWF-BP + fibrinogen & $\mathrm{GPIb}+\alpha_{110} \beta_{3}$ \\
\hline
\end{tabular}

\begin{tabular}{llcc} 
Brightificld / Fluorescence parameters & Range & Normalized \\
P1 & platelet surface area coverage (\%SAC) & $0-66.47$ & $0-10$ \\
P2 & platelet aggregate (\%SAC) & $0-47.84$ & $0-10$ \\
\hline P3 & thrombus morphological score & $0-5$ & $0-10$ \\
P4 & thrombus multilayer score & $0-3$ & $0-10$ \\
\hline P5 & thrombus contraction score & $0-3$ & $0-10$ \\
P6 & PS exposure (\%SAC) & $0-22.71$ & $0-10$ \\
P7 & secretion (P-selectin positive, \%SAC) & $0-63.44$ & $0-10$ \\
P8 & integrin $\alpha_{\text {IIb }} \beta_{3}$ activation (\%SAC) & $0-48.05$ & $0-10$ \\
\hline
\end{tabular}

\section{Flow cytometry activation (A), secretion (Sec), integrin $\alpha_{\text {mlt }} \beta_{3}$ activation (Int)}

\begin{tabular}{lll} 
A1 & unstimulated & A1-Sec, A1-Int \\
A2 & ADP-stimulated & A2-Sec, A2-Int \\
\hline A3 & CRP-XL-stimulated & A3-Sec, A3-Int \\
A4 & TRAP-stimulated & A4-Sec, A4-Int
\end{tabular}

*(VWF), von Willebrand factor from plasma. that are associated with quantitative platelet traits (count, mean volume, distribution width of mean volume, and mass or crit). ${ }^{16,17}$ Several of the variants appeared to be linked to alterations in platelet activation tendency, in particular by using flow cytometric assessment of agonistinduced integrin $\alpha_{\mathrm{II}} \beta_{3}$ activation and P-selectin expression (measuring platelet secretion) in the genotyped individuals. Detailed studies revealed single nucleotide variants which associated with altered platelet expression levels of the collagen receptor, glycoprotein (GP)VI, and with altered GPVI-induced activation responses. ${ }^{18-20}$

In the present study we aimed to evaluate, in a cohort of genetically defined healthy subjects, the underlying reasons of inter-individual variability in a microspot-based multiparameter assay of thrombus formation. The results were therefore related to a set of 24 other variables, including hematologic parameters, platelet glycoprotein expression levels and platelet activation markers. Prediction models were built to link specific variables. Blood samples from patients with bleeding disorders were used to interpret the observed relations.

\section{Methods}

A detailed description of the methods is available in the Online Supplement.

\section{Blood donors and blood collection}

Studies were approved by the Maastricht University Medical Center Ethics Committee and the Cambridge East Research Ethics Committee (Genetic analysis of platelets in healthy individuals, REC ref 10/H0304/65). Healthy subjects (laboratory population) in cohort $1(n=10)$ donated three blood samples at 2- to 4-week intervals. Genotyped healthy subjects (cohort $2, n=94$ ) were analyzed in a period of 2 weeks. These subjects were registered in the National Institute for Health Research (NIHR) BioResource (Unicorn-2 study). Genotyping of subjects in cohort 2 was performed as described elsewhere. ${ }^{20}$ The subjects' demographics are indicated in Online Supplementary Table S1.

Included patients had confirmed Glanzmann thrombasthenia: GT1: ITGA2B c.[2943G>A], [2943G>A], p. (=) (homozygous splice mutation); GT2: ITGA2B c.213C>G; p.P71A, c.2051T>G, p.L684A (compound heterozygous point mutations); GT3: ITGA2B, c.621C>T; p.T176I (homozygous point mutation). Two other patients had a confirmed quantitative delta-storage pool disease (reduced mepacrine capture/release): SPD1, SPD2. All patients and the three day-control subjects had normal blood cell counts (Online Supplementary Data File 1D).

\section{High-throughput microfluidics}

Glass coverslips were coated with three microspots of $0.5 \mu \mathrm{L}$ (3 $\mathrm{mm}$ center-to-center distance) using a high-precision mold. Using two sets of coverslips, a total of six different microspots (M1-6) were applied for whole-blood perfusion (see Table 1). Microfluidics assays were performed as described previously, with minor modifications. Details of the standard operating procedures are provided in the Online Supplement. Post-staining of thrombi was performed with FITC-labeled anti-fibrinogen monoclonal antibody (1:100, Dako, F0111, Santa Clara, CA, USA), Alexa Fluor (AF)568 annexin A5 (1:200, Molecular Probes), and AF647 anti-CD62P monoclonal antibody (1:80, Biolegend, London, UK). Representative brightfield and tri-color fluorescence images were taken with an EVOS-FL microscope (Life Technologies), equipped with GFP, RFP and Cy5 dichroic cubes 
and an Olympus UPLSAPO 60x oil-immersion objective. Images were analyzed for the parameters described in Table 1, using semiautomated scripts written in Fiji. Test variability was 5-8\%, depending on the type of surface and parameter.

\section{Platelet immunophenotyping and platelet activation by flow cytometry}

Platelet immunophenotyping and platelet activation were performed basically as described previously. ${ }^{21}$ The platelet activation parameters that were analyzed are presented in Table 1.

\section{Results}

\section{Multiparameter assessment of whole-blood thrombus formation on microspots under flow}

High-throughput microfluidics has been used for indepth characterization of platelet dysfunction in patients with bleeding disorders. The technique uses microspotcoated flow chambers for multiparameter measurement of thrombus formation during whole-blood perfusion at defined wall-shear rates..$^{79}$ In this study, we further standardized this method for inter-subject analysis of platelet function, using blood samples from healthy subjects, with results expected to represent the normal range observed in a population. Procedures included (see also the Online Supplement and de Witt et al..$^{22}$ ): (i) microspot coating using a high-precision mold; (ii) strictly controlled conditions of blood drawing, anticoagulation and storage; (iii) pulse-free blood perfusion through a Maastricht flow chamber at a defined shear rate; (iv) defined time protocols for rinsing, staining and capturing of brightfield and fluorescence microscopic images; (v) pre-defined scripts for consistent analysis of all image sets; (vi) a gallery of exemplary images for the scoring of thrombus parameters; and (vii) comparative analysis by trained personnel performing flow runs and blind image analysis. Out of a list of 52 different surfaces, ${ }^{7}$ we selected six microspots (M1-6) to provide the most discriminative information on small changes in thrombus formation. For each surface, eight outcome parameters $(P 1-8)$ were defined, as indicated in Table 1.

Employing this standardized procedure, after wholeblood perfusion over each of the microspots, representative images were taken to visualize and assess: platelet adhesion $(P 1)$, platelet aggregation and thrombus morphology (P2-5); platelet phosphatidylserine exposure, as a marker of procoagulant activity (P6); P-selectin expression, to measure secretion (P7); and fibrinogen binding, to report on integrin $\alpha_{\mathrm{II}} \beta_{3}$ activation (P8). Image sets for a typical healthy control subject are given in Figure 1A.

Blood perfusion over microspot $M 1$ (collagen type I) resulted in the formation of large thrombi with contracted aggregates of platelets, high in activation markers (phosphatidylserine exposure, P-selectin expression, $\alpha_{\text {III }} \beta_{3}$ activation), linked to a relatively high GPVI signal.' Microspot M2 (collagen type III) produced smaller platelet aggregates with less pronounced activation markers, corresponding to more limited GPVI signaling. Microspot $M 3$ [von Willebrand factor (VWF) + laminin] gave a monolayer of platelets with P-selectin expression and $\alpha_{\text {II }} \beta_{3}$ activation, but essentially no phosphatidylserine exposure, indicative of the primary adhesive role of the laminin receptor, integrin $\alpha_{6} \beta_{1}$. Microspot M4 contained a combination of collagen-derived peptides [VWF-
$\mathrm{BP}+$ GFOGER-(GPO) ] giving similar thrombi as on collagen type I. Microspot M5 (VWF-BP + rhodocytin) also produced full aggregates with contraction of platelets expressing activation markers. Microspot M6 (VWF-BP + fibrinogen) triggered mainly platelet adhesion with the scattered presence of small platelet aggregates, showing limited P-selectin expression and $\alpha_{\mathrm{II}} \beta_{3}$ activation. The observations for $M 1, M 4$ and $M 5$ are in agreement with the capability of GPVI and CLEC-2 adhesive surfaces to support full thrombus formation in flow assays.?

To evaluate the performance of the standardized method, we collected three different blood samples from ten healthy donors (cohort 1), and determined the coefficients of variation for each of the parameters per microspot (coated $M 1, M 2$ and $M 6$ ). For the majority of the parameters, intra-assay variability of duplicate measurements was $5-8 \%$. For microspots $M 1$ and $M 2$, the median intra-individual coefficients of variation over the three bleeds were $15 \%$ and $18 \%$, respectively, which is considered acceptable for whole-blood assays (Figure 1B). For microspot $M 6$, a higher median intra-individual coefficient of variation of $37 \%$ was obtained, likely due to the fact that parameter values on this 'weak' surface are low. This initial analysis indicated that inter-individual coefficients of variation for the microspots were about twice as high as the intra-individual coefficients of variation (Online Supplementary Table S3).

\section{High-throughput platelet phenotyping by multiparameter assessment of thrombus formation in combination with platelet count and platelet activation markers}

Subsequently, thrombus formation was assessed on microspots $M 1-6$ with blood samples from 94 genotyped healthy subjects from the NIHR BioResource (cohort 2, all blood type O). Donors of either sex had a median age of 64 years (Online Supplementary Table S1). Using the same microfluidics device, brightfield and fluorescence images were recorded and analyzed for all eight parameters $\left(P_{1}\right.$ 8) per microspot. Comparison of the inter-individual coefficients of variation for the 94 samples to the intraindividual ones for a subset of ten of these samples indicated, for the parameters of microspots $M 1-3$, approximately 2-4 times higher inter-individual coefficients of variation. This ratio was 2.5-1.5 times higher for the M46 microspots (Figure 2A). Heat mapping of the normalized values per parameter and microspot showed major differences between the 94 blood samples (Figure 2B). Taken together, these data indicated that a substantial part of the measured values contained a subject-dependent component.

For all 94 subjects, parallel measurements were performed to assess: (i) hematologic parameters using a Sysmex XN-1000 analyzer; (ii) expression levels of platelet membrane proteins; and (iii) platelet activation tendencies by flow cytometry. Hematologic parameters included white and red blood cell counts, hematocrit, platelet count, platelet crit, and mean platelet volume. Surface membrane proteins assessed in unstimulated platelets included major adhesive glycoprotein complexes: integrin $\alpha_{\text {III }} \beta_{3}$ (CD41a, CD41b, CD61), GPIb-V-IX (CD42a, CD42b), integrin $\alpha_{2} \beta_{1}(C D 29, C D 49 b)$, GPIV (CD36), GPVI and the surface-expressed protein tyrosine phosphatase (CD148). Platelet activation tendency (integrin activation and $\alpha$-granule secretion) was measured 
following stimulation via $\mathrm{P}_{2} \mathrm{Y}_{1 / 12}$ receptors (with $\mathrm{ADP}$ ), GPVI (CRP-XL), or the PAR1 thrombin receptor (TRAP) in the presence of aspirin and apyrase (where appropriate), to suppress autocrine-dependent secondary activation. For these platelet traits, heat mapping of the scaled values again showed major inter-individual differences (Figure 2C), similarly as previously described. ${ }^{23}$
Comparative analysis of high-throughput parameters

For systematic evaluation of the eight parameters of thrombi formed on six microspots for the 94 subjects, we performed a correlational analysis across all microspot and parameter combinations. As shown in Figure 3A,B, the majority of parameters tended to correlate positively per microspot, suggesting a 'thrombus profile' represented by multiple parameters (median $P$ values for all 6 microspots

A

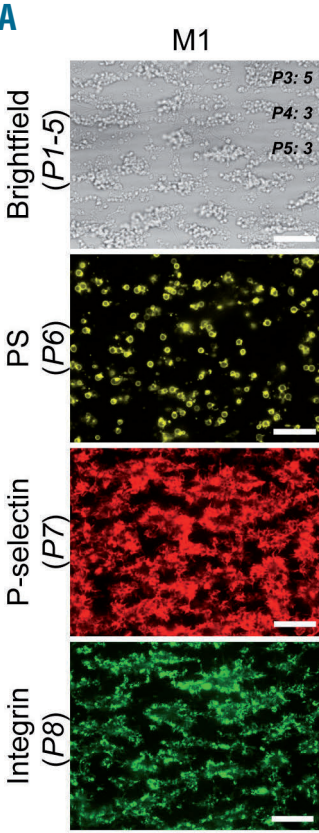

Collagen I
$\mathrm{M} 2$

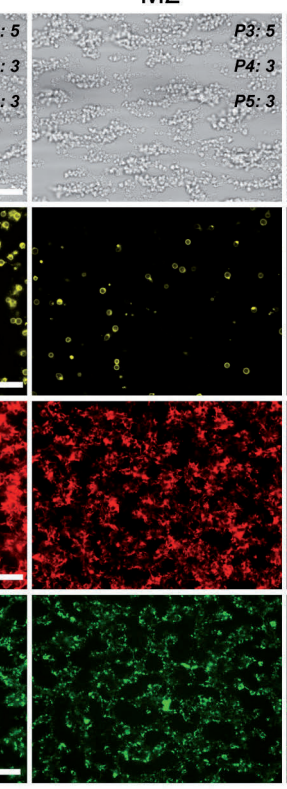

Collagen III
M3

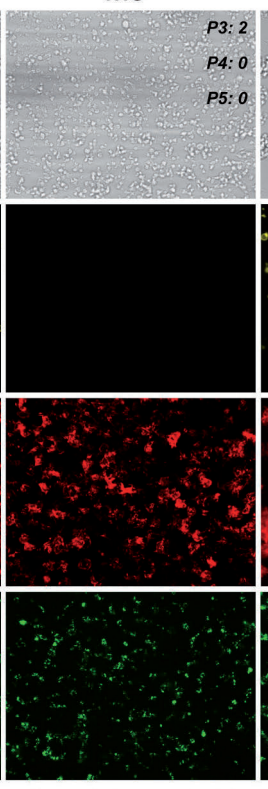

Laminin + VWF

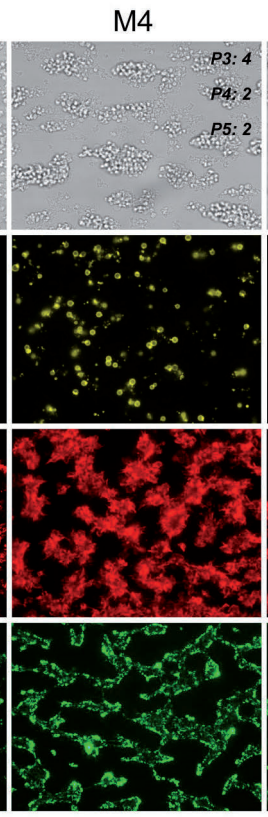

GFOGER-(GPO)
M5

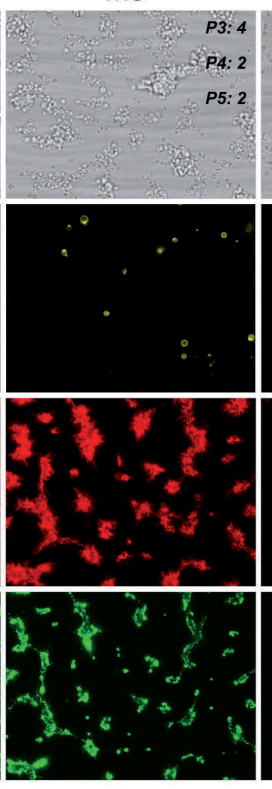

Rhodocytin

+ VWF-BP
M6

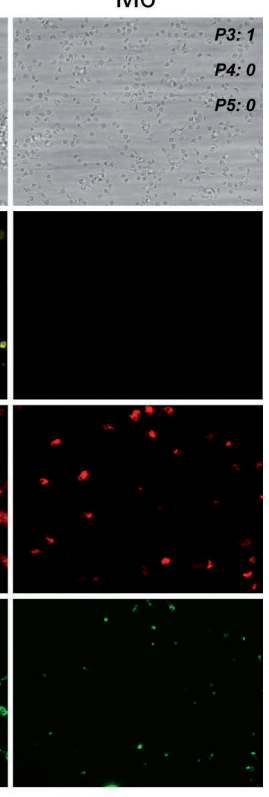

Fibrinogen

+ VWF-BP

B

Inter-individual variation

Intra-individual variation
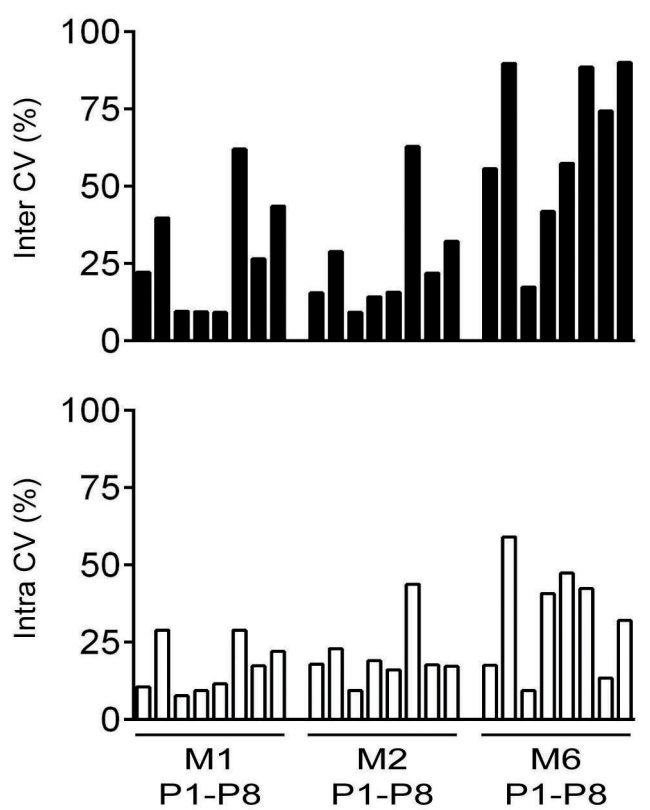

Figure 1. Microscopic imaging of platelet thrombus formation on six different microspots and variability analysis. (A) Representative images after flow of whole blood from a representative healthy subject over series of microspots M1-6 (composition as indicated). For the brightfield images, scored values are indicated for parameters P3 (thrombus morphological score), P4 (thrombus multilayer score), and P5 (thrombus contraction score). Bars, $20 \mu \mathrm{m}$. The definition of all parameters is given in Table 1. (B) Three separate blood samples from ten healthy subjects (cohort 1), taken at intervals of 2-4 weeks, were used to assess thrombus formation on microspots $M 1, M 2$, and $M 6$. Intra- and inter-individual coefficients of variance (CV) are plotted per microspot and parameter. PS: phosphatidylserine. 
$5.22 \times 10^{-5}$ ). Furthermore, positive correlations were also present across multiple microspots. For instance, parameters P2-5 (indicative of thrombus morphology and platelet aggregation) correlated between $M 1$ (collagen I) or $M 2$ (collagen III) with M4 [GFOGER-(GPO) ${ }_{n}+$ VWF-BP] and M5 (rhodocytin + VWF-BP), i.e. all GPVI- and CLEC-2activating surfaces ( $P 2-5$; median $P$ values $4.39 \times 10^{-22}$ ). Furthermore, several parameters (P2-8) correlated between M2 (collagen III) and M3 (laminin + VWF, $\left.P=0.44-1.04 \times 10^{-11}\right)$. Typically, parameters for microspot
M6 (fibrinogen + VWF-BP) showed no more than poor correlation with those of other surfaces.

Multiparameter correlation and regression analysis were also performed to compare subjects' age and sex, hematologic data, platelet glycoprotein expression levels and agonist-induced platelet activation markers by flow cytometry. The interaction matrices displayed in Figure 3C,D show the expected correlation of sex (much stronger than age) with red blood cell and platelet counts $(P<0.014)$, but not with other traits. Subjects' age correlated weakly with
A

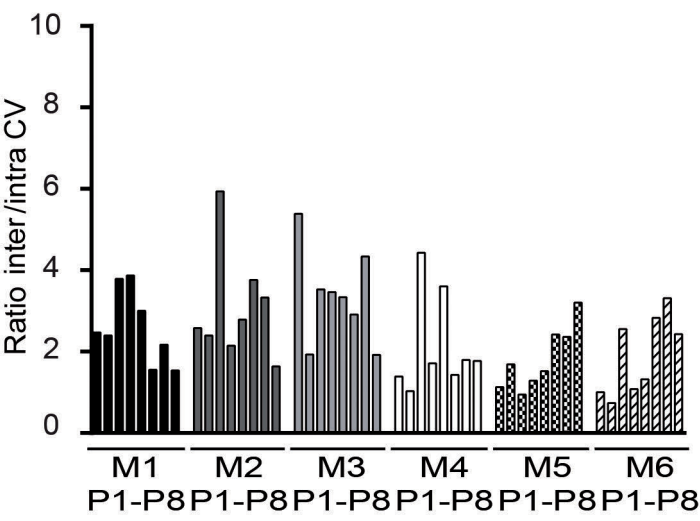

C Normalized values

0246810 value

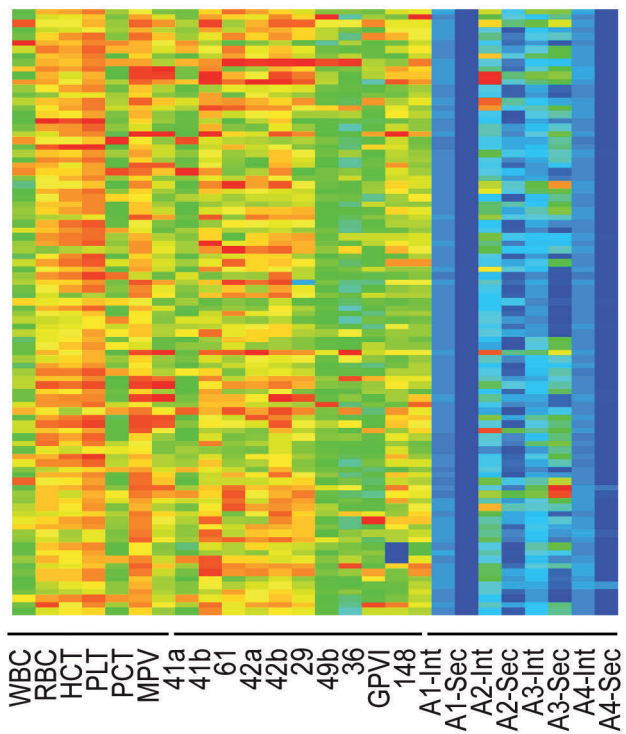

Subject 1
Normalized values
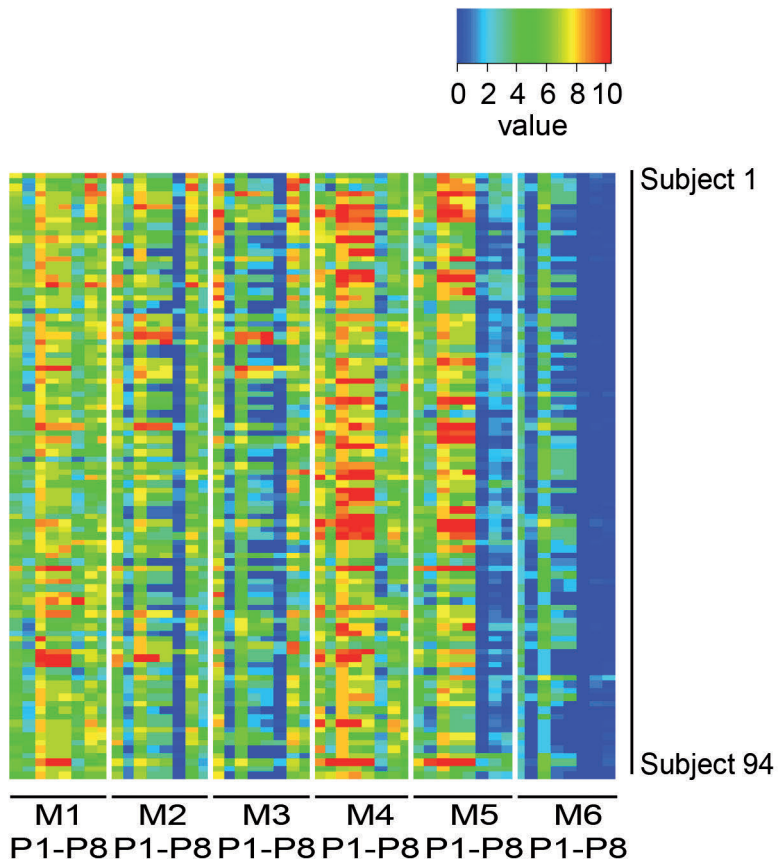

P1-P8P1-P8P1-P8P1-P8P1-P8P1-P8
Subject 94

Figure 2. Inter-subject differences in platelet thrombus formation and other platelet traits. (A) Using blood samples from 94 genotyped healthy subjects (cohort 2), parameters of thrombus formation were assessed on microspots M16. Duplicate samples were analyzed for ten of these subjects. The ratios of interversus intra-individual coefficients of variance per parameter and microspot are shown. (B) Heatmap of normalized parameters per microspot and per subject (rows). Scaling of 0-10 was performed per parameter across all surfaces. (C) Heatmap of additional platelet traits of the cohort of 94 subjects (scaling 0-10). Hematologic variables: white blood cell count (WBC), red blood cell count (RBC), hematocrit $(\mathrm{HCT})$, platelet count (PLT), platelet crit (PCT = count $x$ size), mean platelet volume (MPV); platelet glycoprotein expression (integrin $\alpha_{110} \beta_{3}$ : CD41a, CD41b, CD61; GPIb-V-IX: CD42a, CD42b; integrin $\alpha_{2} \beta_{1}$ : CD29, CD49b; CD36; GPVI; CD148). In addition, activation markers of unstimulated, ADP-, CRP-XL- or TRAP-stimulated platelets (A1-4), regarding integrin $\alpha_{\| 1} \beta_{3}$ activation (Int) and secretion (P-selectin expression, Sec). For details of coding of microspots $(M)$, parameters $(P)$ and activation markers $(A)$, see Table 1. Raw data are provided in Online Supplementary Data File $1 A-C$. 
platelet integrin activation. As required, red blood cell count versus hematocrit, and platelet count versus platelet crit (i.e. count $x$ platelet size), were highly dependent variables $\left(P<5.67 \times 10^{-14}\right)$. Platelet size (mean platelet volume) correlated positively with the expression levels of surface glycoproteins $\left(P=0.03-1.92 \times 10^{-14}\right)$. In addition, the majority of surface protein expression levels correlated signifi-

A

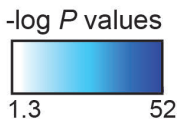

M1 P1-8 M2 P1-8 M3 P1-8 M4 P1-8 M5 P1-8 M6 P1-8

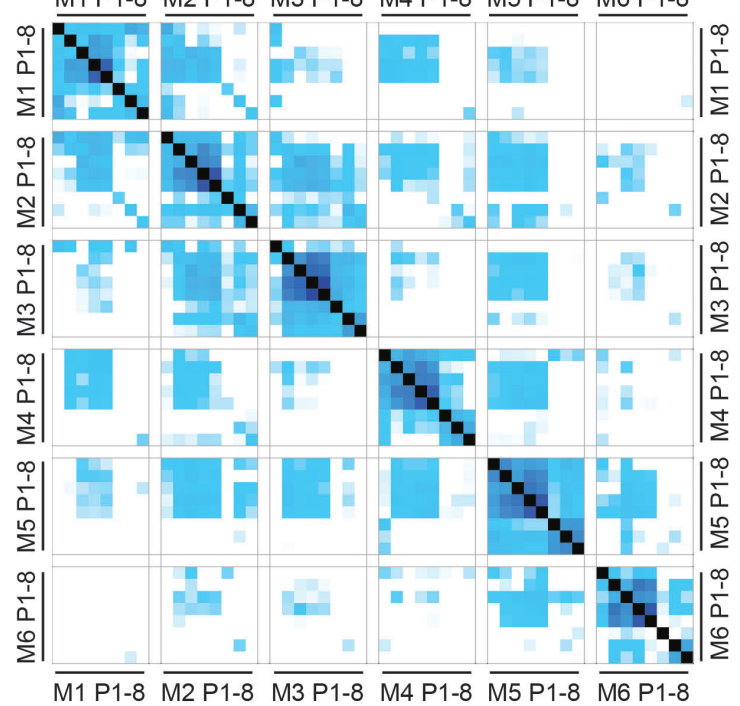

C

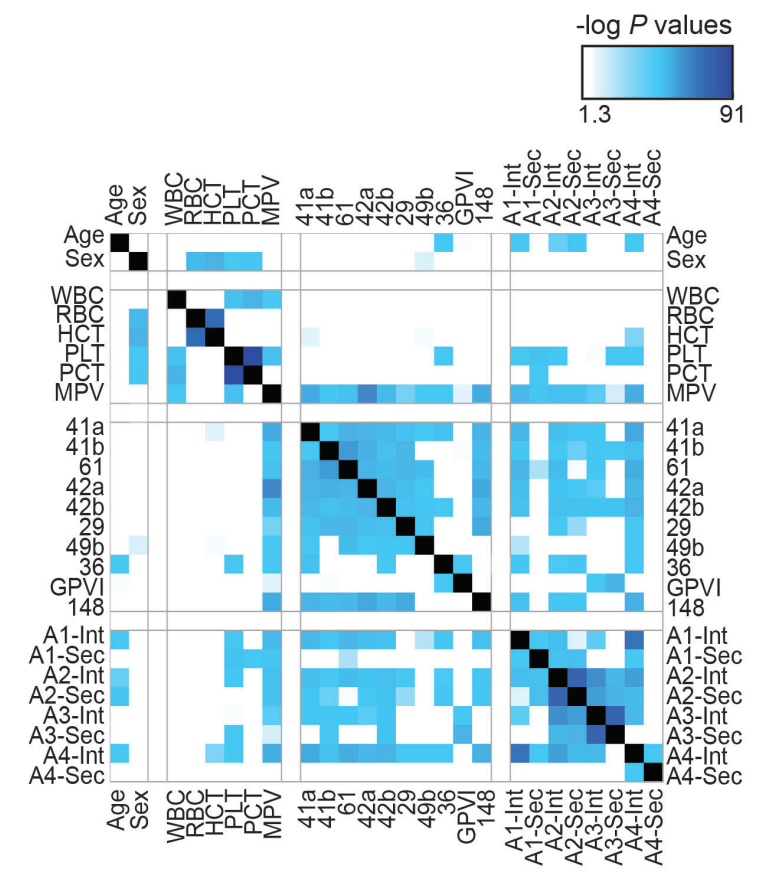

cantly with the components of glycoprotein complexes, integrin $\alpha_{\mathrm{II}} \beta_{3}(\mathrm{CD} 41 \mathrm{a}, \mathrm{CD} 41 \mathrm{~b}, \mathrm{CD} 61), \mathrm{GPIb}-\mathrm{V}-\mathrm{IX}(\mathrm{CD} 42 \mathrm{a}$, CD 42b) and integrin $\alpha_{2} \beta_{1}$ (CD29, CD 49b) $(P=0.012-6.84 \mathrm{x}$ $\left.10^{-11}\right)$. The exception was GPVI, which showed a lower level of correlation with other glycoproteins.

Regression analysis of the markers of agonist-induced platelet activation identified associations between integrin

\section{B}
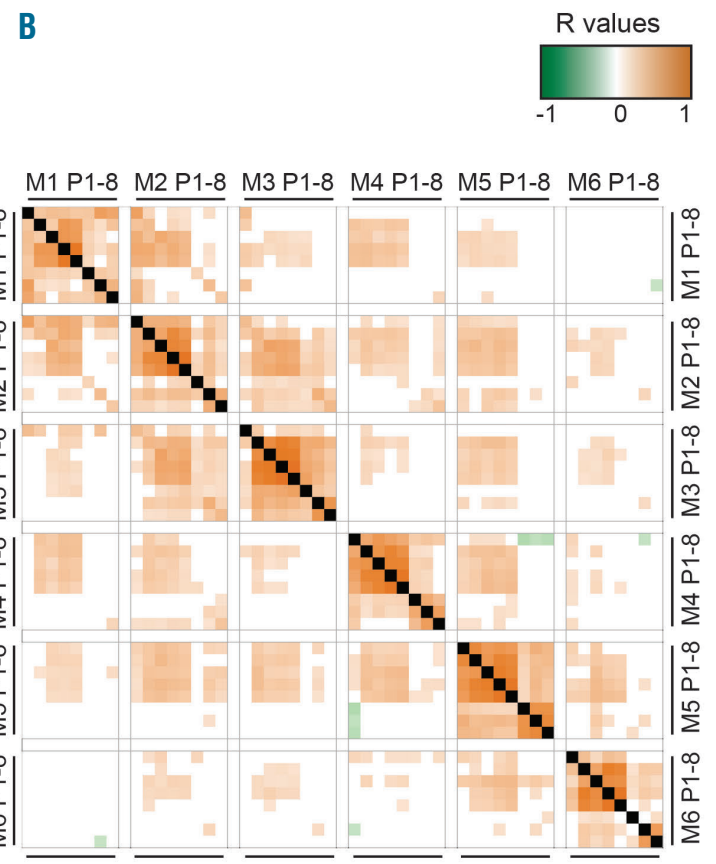

$\overline{\mathrm{M} 1 \mathrm{P} 1-8} \overline{\mathrm{M} 2 \mathrm{P} 1-8} \overline{\mathrm{M} 3 \mathrm{P} 1-8} \overline{\mathrm{M} 4 \mathrm{P} 1-8} \overline{\mathrm{M} 5 \mathrm{P} 1-8} \overline{\mathrm{M} 6 \mathrm{P} 1-8}$
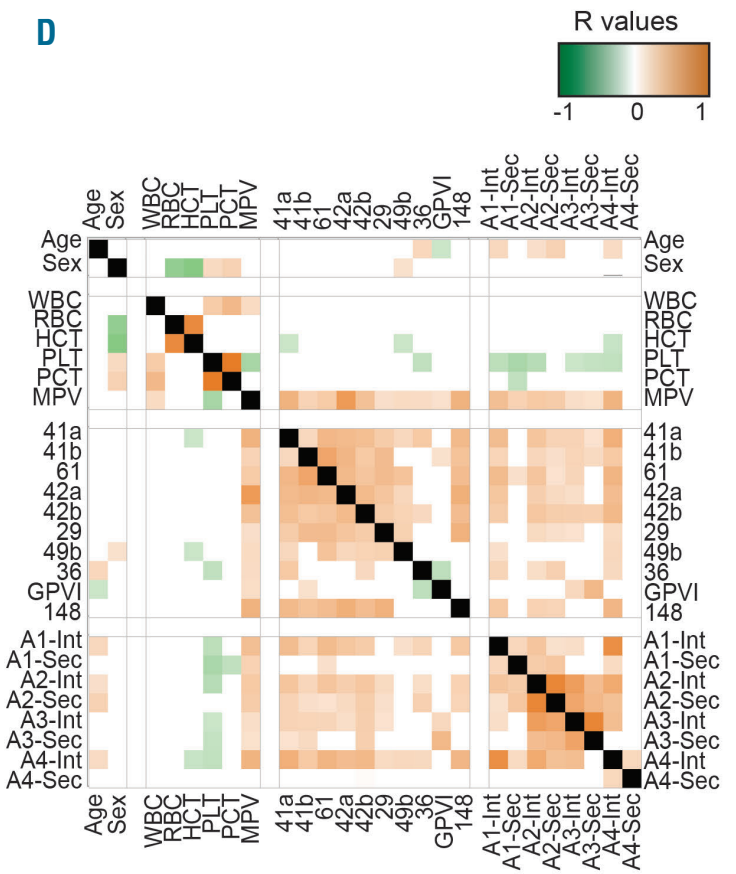

Figure 3. Interactions between parameters of thrombus formation, hematology, platelet surface proteins and activation markers. For 94 genotyped healthy subjects (cohort 2), multiple quantitative traits of thrombus formation, blood cell and platelet parameters, and platelet activation tendency were compared by regression analysis. For coding of microspots $(M)$, parameters $(P)$ and activation markers $(A)$, see Table 1. Other abbreviations are explained in Figure 2. (A, B) Correlation matrices for parameters of thrombus formation. (A) Heat mapped $-\log P$ values, in which dark colors indicate highly significant correlations (white offset at $P=0.05)$. (B) Heat mapped Pearson correlation coefficients $R$, in which dark colors indicate high positive or negative correlations. (C, D) Correlation matrices for age, sex and platelet quantitative traits (hematology variables, platelet glycoprotein expression levels and platelet activation markers). (C) Heat mapped -log $P$ values, with colors as in panel A. (D) Heat mapped Pearson correlation coefficients $R$, with colors as in panel C. Full statistical data are provided in Online Supplementary Data File $2 A-D$. 
activation and secretion in response to either $\mathrm{ADP}(A 2)$ or CRP-XL (A3) and, to a lesser extent, in response to TRAP (A4) (Figure 3C,D). A clear relationship was present between GPVI expression level and CRP-XL-induced activation markers $(P=0.010$ for integrin activation; $P<0.0001$ for secretion). Furthermore, except for TRAP-induced secretion, all platelet activation markers were associated with glycoprotein expression levels (median $P=0.010$ ) and, to a certain extent, with platelet size. Taken together, these results pointed to a subject-dependent component of platelet size and activation, independent of the type of agonist.

\section{Comparative analysis of parameters of thrombus formation with other platelet traits}

Principle component analysis (PCA), using the dataset of 94 subjects, was performed to further analyze the relation- ships between the parameters of thrombus formation and measured platelet quantitative traits. As a way to visualize the results, the relative contributions of components 1 and 2 (C1, C2) were heat mapped for each of the microspots and parameters. The resulting heatmaps (Figure 4) can be read as indicating the parameters of thrombus formation that tend to cluster together per component (in dark color), when compared to other sets of variables.

PCA was first applied to compare parameters $P 1-8$ for each microspot with subjects' age and sex (Figure 4A). Component $1(91.1 \%)$ showed a high correlation for all microspots regarding the parameters $P 1$ (platelet adhesion) and $P 2-5$ (collectively reflecting platelet aggregation and thrombus morphology). Neither age nor sex contributed to this component. Component 2 (1.5\%) showed a cluster of the platelet activation parameters $P 6-8$ (procoagulant activity, secretion and integrin activation). Only
A

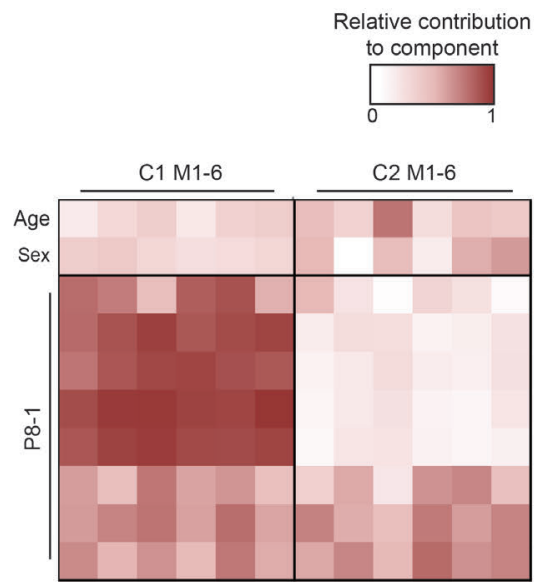

C

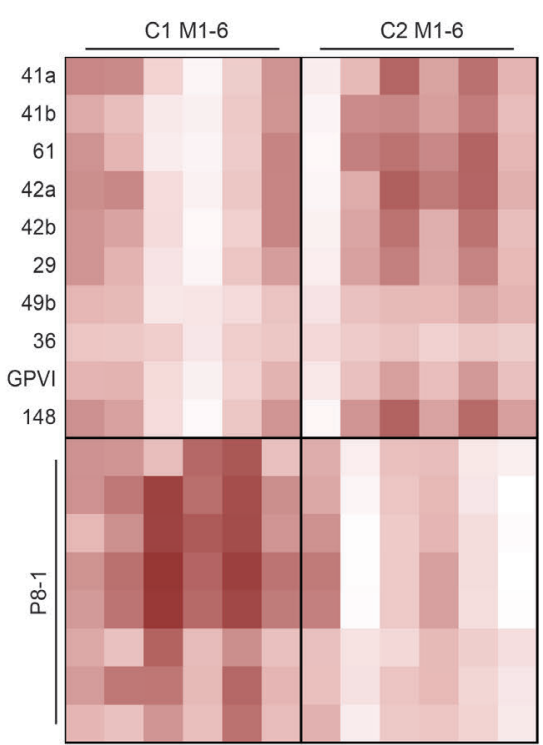

B

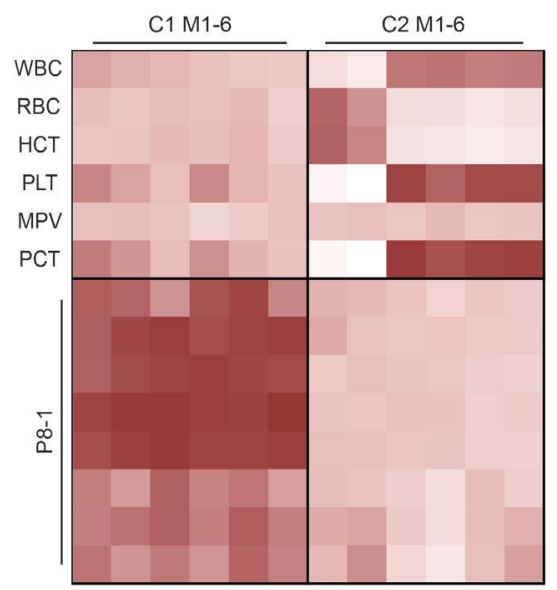

D

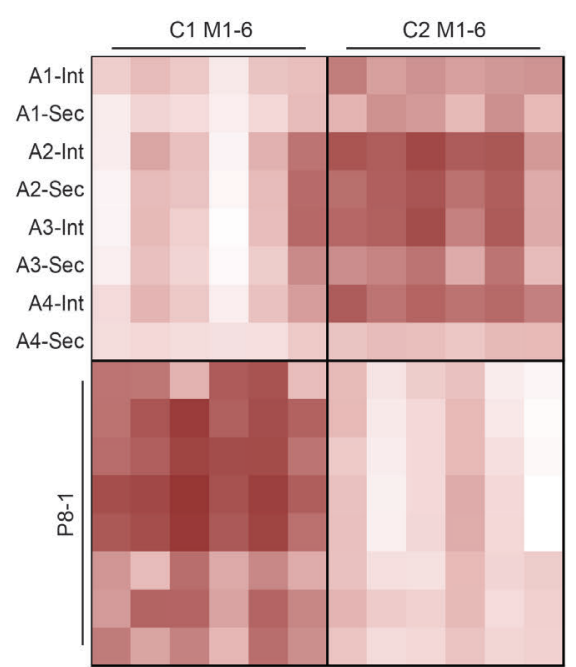

$E$

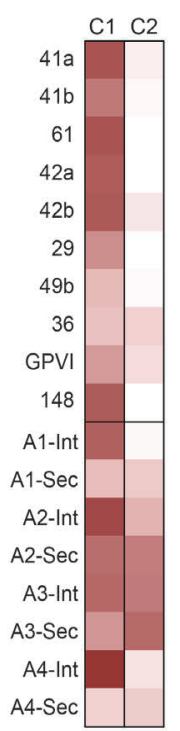

Figure 4. Principal component analysis to reveal correlations between variables of thrombus formation and quantitative platelet traits. Principle component analyses (PCA) of mean centered data from 94 healthy subjects (cohort 2), after univariate scaling as represented in Figure 2B,C. In order to reveal patterns of jointly contributing factors to thrombus formation, scaled data from six microspots (M1-6) and eight parameters (P1-8) were combined in a PCA with sets of other variables from the 94 subjects. Heatmaps show relative contributions of each of the parameters to the first two components, $\mathrm{C} 1$ and $\mathrm{C} 2$. For coding of microspots ( $M$ ), parameters $(P)$, and platelet activation markers (A), see Table 1. (A) PCA of the $M \times P$ matrix in combination with subjects' age and sex. (B) PCA combined with hematologic variables. (C) PCA combined with glycoprotein surface expression levels. (D) PCA combined with integrin $\alpha_{11} \beta_{3}$ activation and secretion markers of agonist-stimulated platelets. (E) PCA of only activation markers of agonist-stimulated platelets with glycoprotein surface expression levels. Color bars of relative contributions to C1 and $\mathrm{C} 2$, ranging from 0 to 1 . White boxes indicate no relation, while dark red boxes indicate a large contribution for the indicated parameters. Raw data are presented in Online Supplementary Data File 2E. 
for microspot $M 3$, did the subjects' age, but not sex contribute to component 2 . Together, these results underscore the earlier conclusion that parameters of platelet aggregation across microspots covariate.

Combined PCA of microspot parameters and hematologic parameters (Figure 4B) resulted in a similar configuration of component $1(97.5 \%)$ as above, with in particular high contributions of microspots $M 1-6$ for P2-5. Platelet count and platelet crit appeared in component 1 for microspots $M 1-2$, while these variables contributed to component $2(1.1 \%)$ for $M 3-6$.

Combined PCA of microspot parameters and glycoprotein expression levels (Figure 4C) revealed a marked contribution of most CD variables (although not of CD36) to the first component (95.5\%) regarding $M 1, M 2$ and $M 6$, suggesting that these expression levels were relevant to thrombus formation on these microspots. Component 2 $(0.8 \%)$ contained most of the glycoprotein expression levels, particularly on $M 3$ and $M 5$ spots.

The combined PCA of microspot parameters with agonist-induced platelet secretion and integrin activation (Figure 4D) revealed a component 1 (89.0\%), again showing large contributions for $M 1-5$ on parameters $P 1-5$ (platelet adhesion, aggregation and thrombus morphology). For microspot $M 6$, component 1 also included the platelet activation markers (A2-4, i.e., ADP, CRP-XL and TRAP). Interestingly, component $2(1.9 \%)$ revealed large contributions of the platelet activation parameters on all other microspots M1-5. These results suggested that microspot M6 (VWF-BP + fibrinogen) is the most sensitive to variation in common agonist-induced platelet activation traits, regardless of the agonist. A final PCA, combining glycoprotein expression markers with platelet activation markers, revealed strong correlations for the variables linked to integrin $\alpha_{\text {II }} \beta_{3}$ (Figure 4E).

Together, these results suggested that a considerable part of the variability in thrombus outcomes between the 94 subjects was linked, in a surface-dependent way, to hematologic traits (platelet count and crit), expression patterns of glycoproteins and to agonist-induced secretion and integrin $\alpha_{\mathrm{II}} \beta_{3}$ activation. Another part seemed to be linked, across surfaces, to platelet aggregation and thrombus morphology.

\section{Prediction models of platelet traits contributing to variation in thrombus formation}

Based on the PCA, predictive models were built to identify the quantitative platelet traits that correlate with thrombus formation parameters. In a first set of partial least squares (PLS) models, we aimed to find the covariance between the individual thrombus parameters and the other platelet parameters. By predicting one variable of the thrombus microspot - parameters matrix at a time, 14 (of 48) parameters had a reduction in the mean square error of prediction, meaning that they could be predicted more accurately than just using the mean (in cross validation). Eleven of the 14 models appeared to be robust (single component, or improvement with an additional component). Most of these models captured a limited amount of the variation, although, for parameter $P 1$, three models (M4P1, M5P1, M2P7) predicted $>5 \%$ (6-11\%) of the total variation (Figure $5 \mathrm{~A})$. This analysis explained a small part of the variance, although with a focus on platelet adhesion $(P 1)$ across the surfaces. Given the limited biological insight of this effort, additional modeling was performed.
Since thrombus formation on three out of the six microspots (M1, M2, M4) was GPVI-dependent, additional prediction models were made to relate the values of GPVI-induced integrin $\alpha_{\mathrm{II}} \beta_{3}$ activation $(A 3-\operatorname{Int})$ and secretion $(A 3-S e c)$ to the thrombus parameters. All models were checked by leaving-one-out cross-validations. To predict GPVI-induced integrin activation, an orthogonal PLS model was built with two components. This resulted in a $\beta$ matrix for each of the microspots and parameters (Figure $5 \mathrm{~B} i)$. For the collagen microspots $(M 1-2)$, parameters of platelet adhesion and activation $(P 1, P 6-7)$ gave a positive weight to the prediction, as well as the majority of parameters of M6 (VWF-BP + fibrinogen). Interestingly, for the strongest GPVI-dependent surfaces (M1, M4), parameters determining platelet aggregation under flow (P2-5, P8) weighed negatively on the prediction. Concerning GPVI-induced secretion, the most suitable PLS model had a single principal component. The resulting $\beta$ matrix was similar to that predicting GPVI-induced integrin activation, albeit with more negative predictive weights for some M5-6 parameters (Figure 5Bii). Taken together, this analysis of the collagen surfaces indicates a positive relationship between GPVI-induced platelet integrin activation/secretion (assessed by flow cytometry) and platelet adhesion and activation in a thrombus.

\section{Prediction of genetic variants of platelet proteins associated with variation in thrombus formation}

Genome-wide association studies have identified several hundreds of genetic variants associated with quantitative platelets traits. ${ }^{16}$ Epigenetic mapping has revealed that many of these variants are regulatory and lie within superenhancer regions that are implicated in megakaryocyte differentiation and platelet production..$^{20}$ For three variants associated with platelet traits, we analyzed whether these were also associated with thrombus formation parameters. Univariate linear regression analysis for the whole $M$ $P$ matrix was used to identify associations with the following single nucleotide variants: rs1613662 (GP6), rs3557 (FCER1G) and rs2363877 (VWF-CD9) (Figure 5C).

The single nucleotide variant rs1613662 is a non-synonymous variant in the GP6 gene, while rs3557 is located in the 3' untranslated region of FCER1G (FC $\gamma$ receptor chain, a co-receptor of GPVI) in a megakaryocytic superenhancer region. Subjects carrying the major allele of either variant (rs1613662, AA; and rs3557, TT) have higher levels of platelet GPVI, and higher CRP-XL-induced platelet activation. ${ }^{20}$ For the present data set, regression analysis indicated inter-allelic differences, in the same direction, in thrombus formation parameters at the collagen surfaces $M 1-2$ for $P_{1}$ (platelet adhesion) with rs1613662 (Figure 5C $i$, ii). In addition, for rs3557 we identified associations at M1-2 and M4, i.e., the other GPVIdependent surface, regarding P8 (integrin activation). Unexpectedly, alleles of both variants were also associated with parameters at the CLEC-2-dependent microspot $M 5$ (VWF-BP + rhodocytin).

The variant rs2363877 is located in a megakaryocytespecific super-enhancer site that interacts with the gene promoters of VWF and CD9. This single nucleotide variant is linked to opposite changes in expression levels of platelet-stored VWF and the surface levels of the tetraspanin CD9. ${ }^{20}$ Here an allelic association was identified at microspot M5 for secretion (P7) (Figure 5Ciii). Trends were also seen for platelet adhesion $(P 1)$ at the 
microspots $M 1$ and $M 5$. Interestingly, the rs2363877 allele linked to increased thrombus parameters at $M 1$ was also associated with high CD9 levels, but low platelet VWF levels. ${ }^{24}$ Altogether, these analyses identified novel variation in platelet thrombus parameters associated with GP6 signaling for GPVI/collagen-dependent platelet adhesion $(P 1)$ and activation $(P 6-8)$.

\section{Thrombus signature based on parameters of platelet aggregation}

To further evaluate the inter-subject differences in thrombus formation, blood was obtained from three patients with Glanzmann thrombasthenia (confirmed mutations in ITG2B, defective integrin $\alpha_{\mathrm{II}} \beta_{3}$ expression), two patients with delta storage pool disease (reduced dense granule secretion), and three healthy day-control subjects. Application of the multiparameter test with the patients' blood samples revealed identifiable patterns of altered thrombus formation on all microspots (raw data in
Data File 1D). Interestingly, the altered patterns seen with the three Glanzmann samples (Online Supplementary Figure $S 1 A-C$ ) reinforced the earlier PCA results that, across microspots, the values of P2-5 (and to a lesser extent P8) tended to cluster. All of these parameters relate to platelet aggregation and contraction, i.e. platelet functions known to be impaired in these patients. For the patients with storage pool disease, similar but less striking patterns of changes were observed (Online Supplementary Figure $S 1 D, E)$.

Subsequently, we determined whether this cluster of parameters also provides information on the thrombus signature for healthy subjects. The summative scaled value $\Sigma(P 2-5, P 8)$ was calculated per microspot and for all microspots. Supervised clustering, ranking the 94 subjects according to this summation, did indeed point to patterns of high and low platelet aggregation, which extended over multiple microspots (Figure 6A). For the three Glanzmann patients, these summative values were very low, as

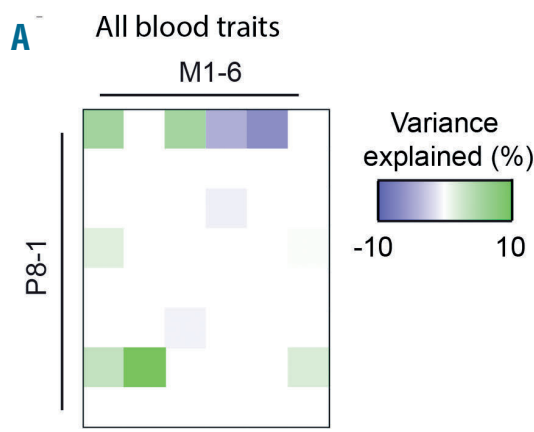

B

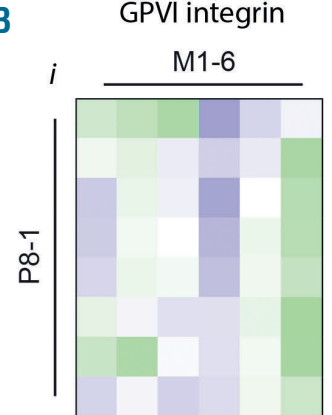

C

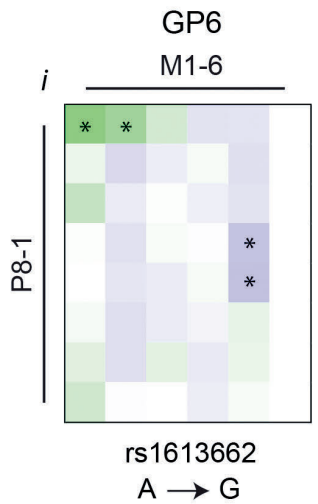

GPVI secretion

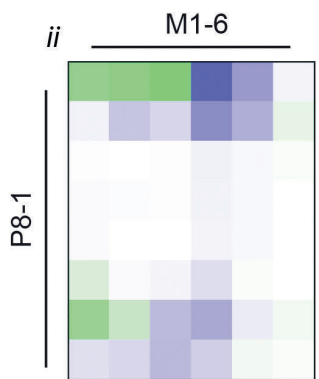

Beta values

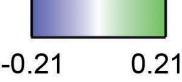

Figure 5. Prediction models explaining variation in thrombus formation. (A) Partial least squares (PLS) models determining the covariance for each of the individual thrombus parameters (M1-M6, P1-P8) and all other platelet traits. Fourteen (from 48) parameters showed a relevant prediction, capturing $0.2-10.9 \%$ (blue color intensity) of the variation. (B) Beta matrix per individual parameter for the PLS models of GPVI-induced platelet activation (unit variance scaled, mean centered data): (i) GPVIinduced $\alpha_{u} \beta_{3}$ activation (A3-Int, 2 components orthogonal PLS); (ii) GPVI-induced secretion (A3-Sec, 1 component PLS). Positive and negative weights are indicated by different colors. (C) Matrix of significance per parameter (quantile normalized linear regression, and likelihood ratio test per allelic score), expressed as $P$ values, for the following genetic variants: (i) GP6, rs1613662 (AA, GA, GG; $\mathrm{n}=63,29$, 1); (ii) FCER1G, rs3557 (TT, TG, GG; $\mathrm{n}=67,24,2$ ); (iii) VWF-CD9, rs2363877 (GG, GA, AA $\mathrm{n}=20,47,26$ ). Significance is indicated by green color intensity and $* P<0.05$.
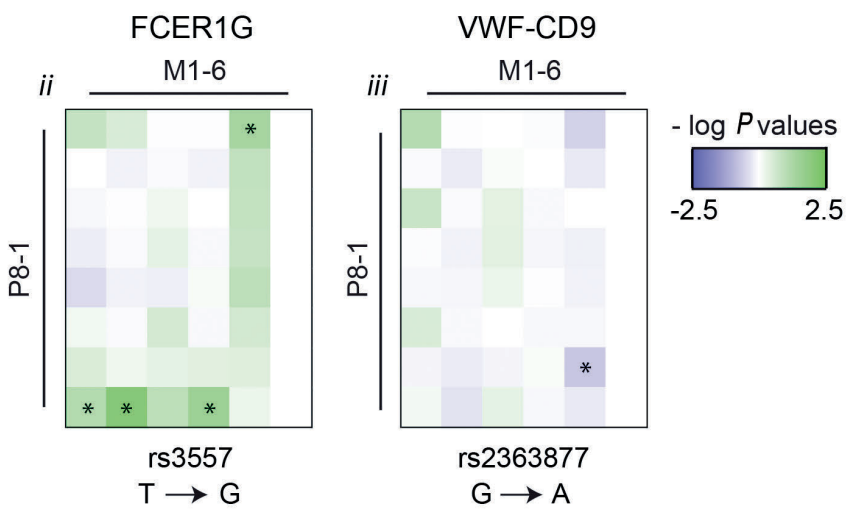
expected. For the two patients with storage pool disease, these values appeared to be in the lower ranges of normal.

To further assess the subject-dependent component of this thrombus signature, we evaluated the intra- and interindividual variations in $\Sigma(P 2-5, P 8)$. This analysis indicated 2 to 5 times higher inter-individual coefficients of variation (Figure 6B). Interestingly, this measure of the thrombus signature was not related to GPVI expression of GPVIinduced platelet responses, thus pointing to other factors determining the platelet aggregation profile under flow conditions.

\section{Discussion}

Multiparameter assessment of thrombus formation using microfluidic assays has proven to be relevant for the assessment of platelet dysfunction in patients with different bleeding diatheses. ${ }^{7-9}$ In the present study, we further developed this technique to assess the sources of variability in thrombus formation between individuals with normal hemostasis. We evaluated platelet quantitative traits as well as thrombus formation in a cohort of 94 genetically defined healthy subjects. Regression analysis identified an overall strong correlation between most parameters per microspot surface. When comparing different microspots, especially parameters of platelet aggregation and throm- bus morphology (P2-5) correlated with each other for GPVI- and CLEC-2-activating surfaces (M1-2, M4-5). These parameters appear to describe subject-dependent differences in thrombus formation or thrombus signature, such as was also deduced from strong alterations in these parameters in blood from patients with Glanzmann thrombasthenia. Comparative matrices of other traits did not reveal age or sex as determinants of thrombus formation, in accordance with previous analyses. ${ }^{20,25,26}$ A clear relation was however observed between GPVI expression levels and CRP-XL-induced platelet activation markers, which is in agreement with an early report. ${ }^{27}$

Several PCA were applied to compare the matrix of thrombus formation parameters with other platelet traits. An effect of platelet count and crit was seen for thrombus formation on collagen-containing microspots (M1-2), in agreement with an earlier conclusion that platelet count is a regulatory determinant of collagen-dependent thrombus formation. ${ }^{9}$ Regression analysis confirmed the predicted associations between mean platelet volume and expression levels of most platelet membrane glycoproteins. In addition, associations were seen between platelet activation markers after ADP, CRP-XL or TRAP activation and glycoprotein expression levels.

A PCA of thrombus parameters plus glycoprotein expression levels revealed marked contributions of most $\mathrm{CD}$ markers to the first component (95.5\%) for $M 1, M 2$
A

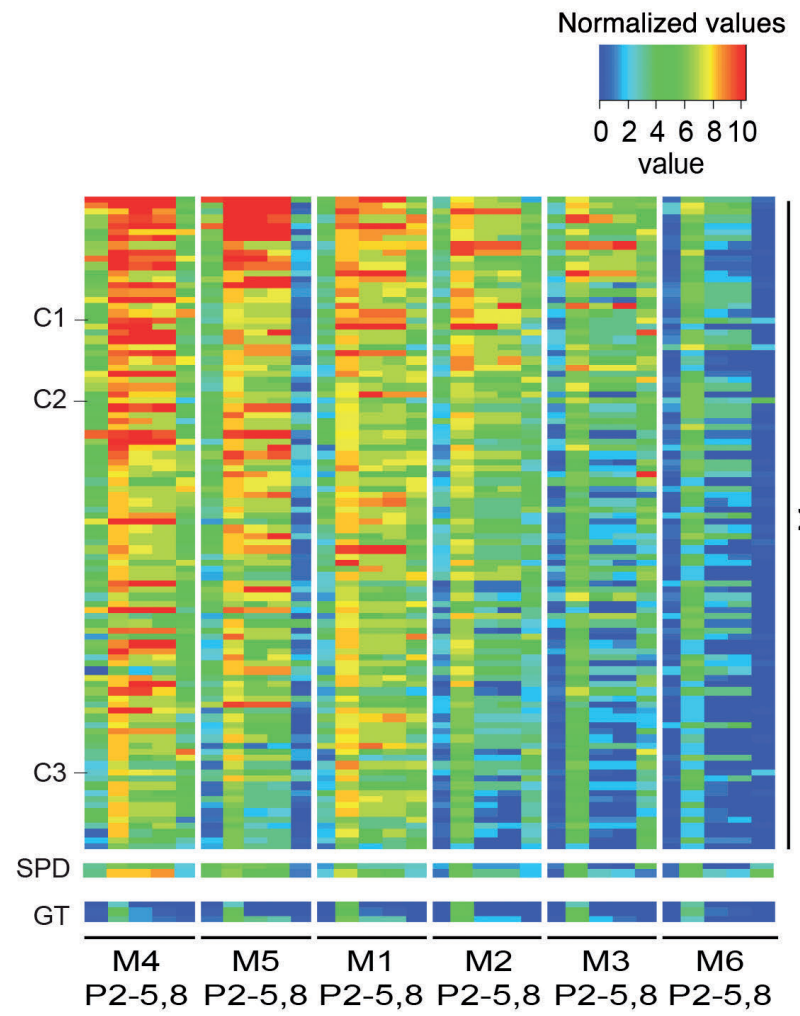

Figure 6. Identification of thrombus signatures across microspots. (A) Supervised clustering of thrombus parameters $P 2-5$ and $P 8$ for microspots M1-6, aligned as indicated. Ranking of data from 94 subjects (cohort 2), patients with storage pool disease (SPD1-2) or Glanzmann thrombasthenia (GT1-3) and day-controls (C1-3) was according to the sum of normalized thrombus parameters of all surfaces $\Sigma(P 2-5, P 8)$. Order of subjects (compare Figure 1B): 92, 49, 33, 4, 57, 35, 9, 19, 6, 8, 15 $24,78,50,16,42,20,65, \mathrm{C} 3,72,41,44,76,71$, $18,25,26,52,40,10, \mathrm{C} 2,51,5,12,28,29,36$ $67,23,32,38,2,88,17,54,59,89,81,60,80$ $11,82,73,79,69,55,87,48,47,66,53,7,70$, $93,64,1,45,46,30,91,3,94,77,68,43,34$ $86,62,58,37,75,63,27,39,83, \mathrm{C} 1,22,85$ $90,21,13,31,61,74,84,56$, SPD1, SPD2, GT1 GT2, GT3. Note the overall consistency of the platelet aggregation-linked parameters $(P 2-5, P 8)$ per subject. (B) Intra- and inter-individual coefficients of variance $(\mathrm{CV})$ of summative value $\Sigma(P 2$ $5, P 8)$ per microspot (cohort 2), together with ratios indicating high subject-dependency of this thrombus signature.

\begin{tabular}{lccccccc}
\hline & M4 & M5 & M1 & M2 & M3 & M6 & M1-6 \\
\hline Intra CV (\%) $\Sigma P$ 2-5,8 & 8.54 & 13.67 & 2.99 & 7.82 & 17.27 & 36.66 & 7.08 \\
Inter CV (\%) $\Sigma$ P2-5,8 & 37.20 & 26.24 & 11.88 & 20.22 & 42.42 & 44.26 & 22.04 \\
Ratio inter/intra & 4.36 & 1.92 & 3.98 & 2.58 & 2.46 & 1.21 & 3.11 \\
\hline
\end{tabular}


and M6, suggesting that these expression levels determine, at least partially, thrombus formation on these microspots. The PCA with platelet activation markers did not reveal clear links with thrombus parameters, except for the weakest surface $M 6$. Together with the relatively high intra-subject variance of this surface, this finding suggests that $M 6$ parameters detect small changes in the activation tendency of platelets, possibly related to the quality of the blood sample.

A PLS prediction model was developed to assess the extent to which the platelet traits can explain the measured inter-subject variation in thrombus formation. Results revealed a limited predictability for platelet adhesion (P1) at various microspots (M4-5). Overall, $1-2 \%$ of the variance could be predicted in this way, likely because of the multivariate and multidirectional nature of these platelet traits. As a more targeted and powerful approach, regression models were built to predict the $M-P$ matrices from subject-dependent values of GPVI-induced integrin activation and secretion. As expected, the CRP-XLinduced integrin activation and secretion measures were positively associated with GPVI-dependent microspot (M1-2) parameters of platelet adhesion and activation ( $P 1$, $P 7)$. A negatively weighted prediction was seen for parameters of M4-5 (VWF-BP + GFOGER-(GPO)n; VWF-BP + rhodocytin), possibly because of the relatively large roles of $\alpha_{2} \beta_{1}$ and CLEC-2, respectively, on these surfaces. Regarding GPVI-induced integrin activation, a positively weighted prediction was seen for thrombi on the $\alpha_{\mathrm{II}} \beta_{3^{-}}$ dependent microspot $M 6$, suggesting that the variation on this surface had different causes.

Regression analysis also indicated associations for single nucleotide variants that are linked to alterations in platelet size or GPVI-induced platelet activation. ${ }^{20,27}$ For two single nucleotide variants linked to GPVI expression, rs1613662 (GP6) and rs3557 (super-enhancer for FCER1G), allelic associations were identified with the GPVI-dependent microspots (M1-2, M4) for platelet adhesion (P1) and platelet activation ( $P 8$, integrin activation) parameters. In addition, single nucleotide variant associations were observed for thrombus formation on the CLEC-2-dependent microspot $M 5$, for unclear reasons. Other authors have used microfluidics assays to show that subjectdependent differences in platelet calcium fluxes contribute to a variation in collagen-dependent thrombus formation. ${ }^{28}$ This work supports our findings of the presence of a subject-dependent factor in GPVI-induced platelet activation and thrombus formation on collagen surfaces.

In contrast, no associations were seen for the single nucleotide variant of the VWF-CD9 locus. Plasma levels of VWF are known to determine the thrombus outcome in flow assays. ${ }^{29}$ However, the variant rs 2363877 only modifies platelet-accumulated VWF, rather than plasma VWF. ${ }^{20}$

A limitation of our study is that, despite the measurement of over 70 different blood and platelet traits, the number of healthy subjects was restricted to 94, thus limiting the statistical power and the precise assignment of the meaning of all individual parameters. Further work in larger cohorts of healthy subjects, and in patients with known platelet and plasma disorders, will add to the characterization of many of these parameters.

\section{Acknowledgments}

Support was obtained from the Cardiovascular Center (HVC), Maastricht University Medical Center, the Center for Translational Molecular Medicine (Incoag/Mikrobat), Interreg Euregio Meuse-Rhin (Polyvalve), the National Institute for Health Research (NIHR) Cambridge Biomedical Research Centre and the National Health Service Blood and Transplant (NHSBT). We gratefully acknowledge the participation of all NIHR BioResource volunteers, and thank the NIHR BioResource center and staff for their contribution. We also thank the National Institute for Health Research and NHS Blood and Transplant for funding support. $K D$ is supported as a HSST trainee by Health Education England. Funders had no role in the study design, data collection and analysis, decision to publish, or preparation of the manuscript.

\section{References}

1. Stegner D, Nieswandt B. Platelet receptor signaling in thrombus formation. J Mol Med (Berl). 2011;89(2):109-121.

2. Versteeg HH, Heemskerk JW, Levi M, Reitsma PH. New fundamentals in hemostasis. Physiol Rev. 2013;93(1):327-358.

3. Swieringa F, Kuijpers MJ, Heemskerk JW, van der Meijden PE. Targeting platelet receptor function in thrombus formation: the risk of bleeding. Blood Rev. 2014;28(1):9-21.

4. Von Hundelshausen P, Agten SM, Eckardt V, et al. Chemokine interactome mapping enables tailored intervention in acute and chronic inflammation. Sci Transl Med. 2017;9(384)

5. Ruggeri ZM. Platelet adhesion under flow. Microcirculation. 2009;16:58-83.

6. Heemskerk JWM, Sakariassen KS, Zwaginga JJ, et al. Collagen surfaces to measure thrombus formation under flow: possibilities for standardization. J Thromb Haemost. 2011;9(4):856-858.

7. De Witt SM, Swieringa F, Cavill R, et al.
Identification of platelet function defects by multi-parameter assessment of thrombus formation. Nat Commun. 2014;5:4257.

8. Mattheij NJ, Braun A, van Kruchten R, et al. Survival protein anoctamin-6 controls multiple platelet responses including phospholipid scrambling, swelling, and protein cleavage. FASEB J. 2016;30(2):727-737.

9. Nagy M, Mastenbroek TG, Mattheij NJ, et al. Variable impairment of platelet functions in patients with severe, genetically linked immune deficiencies. Haematologica. 2018;103(3):540-549.

10. Harrison P, Mackie I, Mumford A, et al. Guidelines for the laboratory investigation of heritable disorders of platelet function. $\mathrm{Br}$ J Haematol. 2011;155(1):30-44.

11. Dawood BB, Lowe GC, Lordkipanidze M, et al. Evaluation of participants with suspected heritable platelet function disorders including recommendation and validation of a streamlined agonist panel. Blood. 2012;120(25):5041-5049.

12. Goodall AH, Appleby J. Flow-cytometric analysis of platelet-membrane glycoprotein expression and platelet activation. Methods
Mol Biol. 2004;272:225-253.

13. Hayward CP, Harrison P, Cattaneo M, Ortel TL, Rao AK. Platelet function analyzer (PFA)-100 closure time in the evaluation of platelet disorders and platelet function. Thromb Haemost. 2006;4(2):312-319.

14. Lordkipanidze M, Lowe GC, Kirkby NS, et al. Characterization of multiple platelet activation pathways in patients with bleeding as a high-throughput screening option: use of 96-well Optimul assay. Blood. 2014;123(8):e11-22.

15. Dovlatova N, Lordkipanidzé M, Lowe GC et al. Evaluation of a whole blood remote platelet function test for the diagnosis of mild bleeding disorders. J Thromb Haemost. 2014;12(5):660-665

16. Gieger C, Radhakrishnan A, Cvejic A, et al. New gene functions in megakaryopoiesis and platelet formation. Nature. 2011;480 (7376):201-207

17. Astle WJ, Elding $\mathrm{H}$, Jiang $\mathrm{T}$, et al. The allelic landscape of human blood cell trait variation and links to common complex disease. Cell. 2016;167(5):1415-1429.

18. Joutsi-Korhonen L, Smethurst PA, Rankin A, 
et al. The low-frequency allele of the platelet collagen signaling receptor glycoprotein $\mathrm{VI}$ is associated with reduced functional responses and expression. Blood. 2003;101(11): 4372-4379.

19. Watkins NA, O'Connor MN, Rankin A, et al. Definition of novel GPVI polymorphisms and major difference in haplotype frequencies between populations by a combination of in-depth exon resequencing and genotyping with tag single nucleotide polymorphisms. J Thromb Haemost. 2006;4(6):11971205.

20. Petersen R, Lambourne JJ, Javierre BM, et al. Platelet function is modified by common sequence variation in megakaryocyte super enhancers. Nat Commun. 2017;8:16058.

21. Chen L, Kostadima M, Martens JH, et al. Transcriptional diversity during lineage commitment of human blood progenitors.
Science. 2014;345(6204):1251033.

22. De Witt S, Swieringa F, Cosemans JM, Heemskerk JW. Thrombus formation on microspotted arrays of thrombogenic surfaces. Nat Protocol Exchange. 2014;2014: 3309\#.

23. Garner SF, Furnell A, Kahan BC, et al. Platelet responses to agonists in a cohort of highly characterised platelet donors are consistent over time. Vox Sang. 2017;112(1):18-24

24. Marx I, Lenting PJ, Adler T, Pendu R, Christophe OD, Denis CV. Correction of bleeding symptoms in von Willebrand factor-deficient mice by liver-expressed von Willebrand factor mutants. Arterioscler Thromb Vasc Biol. 2008;28(3):419-424.

25. Eshel-Green T, Berny MA, Conley RB, McCarty OJ. Effect of sex difference on platelet adhesion, spreading and aggregate formation under flow. Thromb Haemost.
2009;102(5):958-965.

26. Favaloro EJ, Franchini M, Lippi G. Aging hemostasis: changes to laboratory marker of hemostasis as we age. Semin Thromb Hemost. 2014;40(6):621-633.

27. Jones CI, Bray S, Garner SF, Stephens J, et al A functional genomics approach reveals novel quantitative trait loci associated with platelet signaling pathways. Blood. 2009;114(7):1405-1416.

28. Flamm MH, Colace TV, Chatteriee MS, et al Multiscale prediction of patient-specific platelet function under flow. Blood 2012;120(1):190-198.

29. Neeves KB, Onasoga AA, Hansen RR, et al Sources of variability in platelet accumulation on type 1 fibrillar collagen in microfluidic flow assays. PloS one. 2013;8(1): e54680. 